\title{
Single Atom and Cluster Catalysis: The Era of Aberration-corrected Scanning Transmission Electron Microscopy
}

\author{
J. Y. Liu
}

Department of Physics, Arizona State University, Tempe, Arizona 85287, USA.

Many industrial catalysts consist of expensive metals finely dispersed onto high-surface-area and inexpensive supports. The purpose of finely dispersing metal/alloy particles is to efficiently utilize the accessible active centers to convert reactant molecules to the desired final products. Since the interior atoms of metal/alloy particles cannot be accessed by the reactant molecules, reduction of particle sizes significantly increases the total number of surface atoms. In the ultimate limit, the metals/alloys can be atomically dispersed. Recent experimental results have demonstrated that individual atoms dispersed onto appropriate supports can act as catalytically active centers for selected chemical reactions [1].

To develop atomically dispersed metal catalysts, knowledge on the atomic level understanding of the catalyst preparation processes, how atomically dispersed metal/alloy catalysts behave under catalytic reaction conditions, and the interactions between metal atoms/clusters and the support surfaces becomes critical. The recent development of aberration-corrected scanning transmission electron microscopy (STEM) techniques for imaging atoms or molecules and for chemically analyzing clusters or individual atoms of interest [2] makes it possible to unravel the mysteries of heterogeneous catalysts. It is expected that aberration-corrected STEM, together with the development of gas/liquid cells, will play a major role in developing supported catalysts, especially atomically dispersed metal catalysts.

To demonstrate the power of sub-angstrom resolution high-angle annular dark-field (HAADF) imaging technique, Figs. 1-3 show atomically dispersed single atom catalysts (Fig. 1a and 1b), small particle (Fig. 2b) and cluster (Fig. 3) catalysts. Periodic structures of surface adsorbed atoms (Fig. 1c) and layerby-layer growth of secondary phases (Fig. 2a) on nanowires have been investigated. The stability of atomically dispersed metals is critical to developing practical catalysts. Individual metal atoms can be anchored by special surface sites [3] or by surface embedding (replacing the surface cations of the support material) [1]. The Pt and Pd atoms shown in Figs. 1a and 1b were extremely stable under electron beam irradiation because these atoms replaced the surface $\mathrm{Zn}$ atoms of the $\mathrm{ZnO}$ nanowire support, thus forming strong bonding with the sub-surface oxygen anions. With decreasing particle sizes, the surfaces of the support materials may strongly influence the structure of small particles or clusters, thus modifying the nature of the active centers, resulting in change of catalytic performances. Epitaxial growth of metal nanoparticles (Fig. 2b) or layers (Fig. 2a) onto the support surfaces stabilizes supported catalysts [4]. Arrangement of corner atoms of metal nanoparticles (indicated by the block arrow in Fig 2b) and the metal particle-support interfacial structures can be investigated. Under electron irradiation, anchored atoms and epitaxially grown clusters can be stable while un-anchored particles (indicated by the block arrow in Fig. 3) can become highly wobbly and mobile. Synthesis-structureperformance relationships of different supported single atom or cluster catalysts will be discussed [5].

References:

[1] B. T. Qiao et al, Nat. Chem. 3 (2011), p. 634; G. Kyriakou et al, Science 335 (2012), p. 1209; E. Bayram et al, ACS Catal. 9 (2012), p. 1947.

[2] W. Zhou et al, Phys. Rev. Lett. 109 (2012), p. 206803.

[3] JH. Kwak et al, Science 325 (2009), p. 1670. 
[4] J. Liu, ChemCatChem 3 (2011) p. 934.

[5] This research was funded by Arizona State University. We gratefully acknowledge the use of facilities within the LeRoy Eyring Center for Solid State Science at Arizona State University.
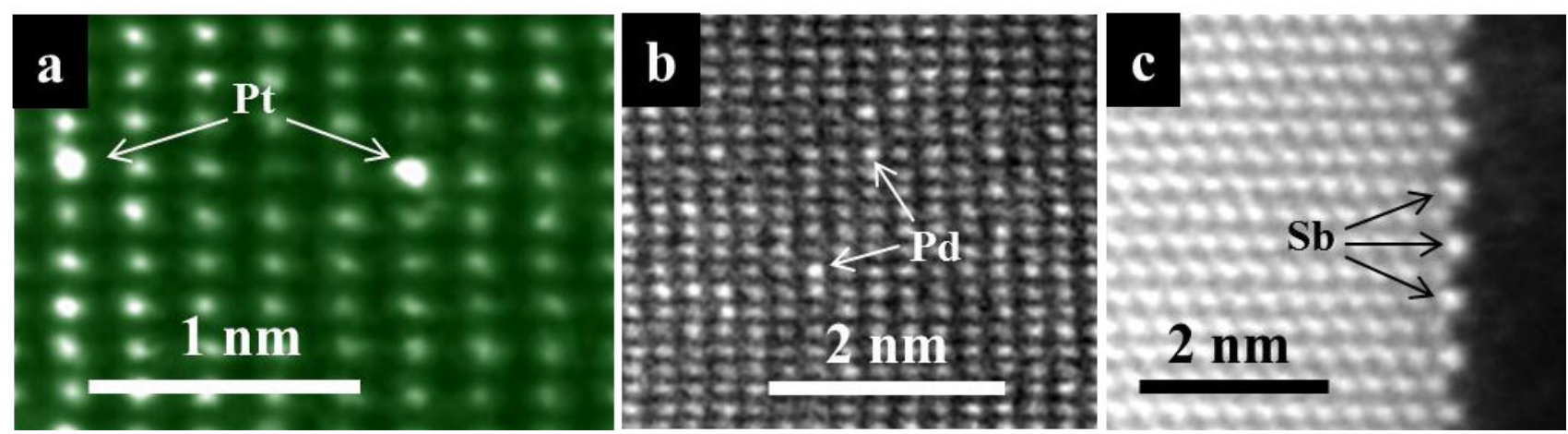

Figure 1. Sub-angstrom resolution HAADF images of individual Pt (a), Pd (b) and columns of Sb atoms (c) dispersed onto the $\{10-10\}$ surfaces of $\mathrm{ZnO}$ nanowires. The $\mathrm{Pt}_{1} / \mathrm{ZnO}$ and $\mathrm{Pd}_{1} / \mathrm{ZnO}$ catalysts are used for reforming of alcohols to produce hydrogen.
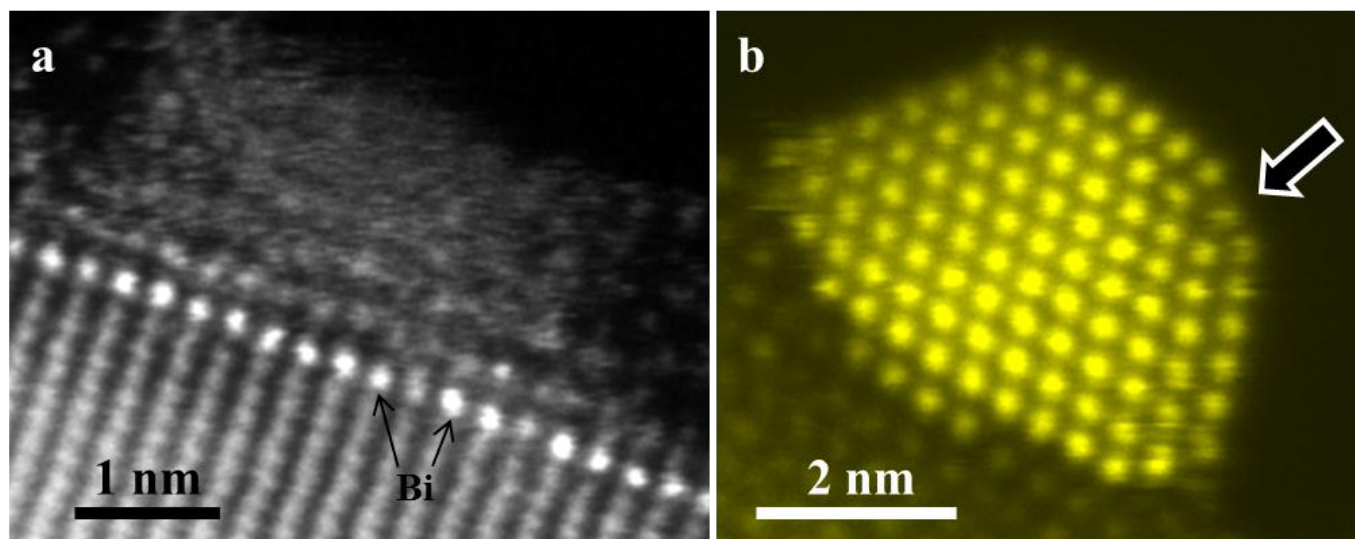

Figure 2. Sub-angstrom resolution HAADF images of a layer of $\mathrm{Bi}$ (a) and an $\mathrm{Au}$ nanoparticle epitaxially grown on a $\mathrm{ZnO}$ nanowire (a) and an $\mathrm{Al}_{2} \mathrm{O}_{3}$ nanorod (b), respectively.

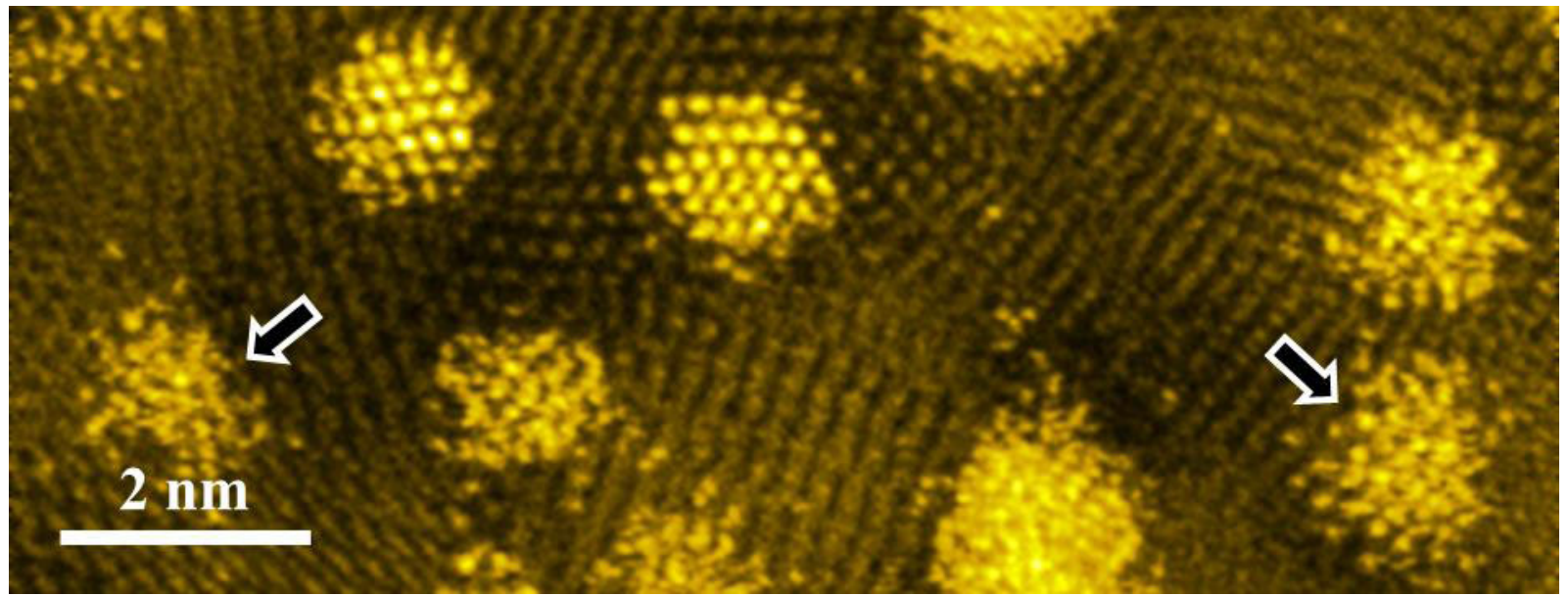

Figure 3. Sub-angstrom resolution HAADF image of individual Pt atoms, clusters and nanoparticles dispersed onto a polycrystalline $\mathrm{ZnO}$ thin film. 